\title{
Finite Element Modeling with Embed Rebar Elements and Steady State Rolling Analysis for Rolling Resistance Test of Pneumatic Tire
}

\author{
Chakrit Suvanjumrat and Ravivat Rugsaj \\ ${ }^{1}$ Department of Mechanical Engineering, Faculty of Engineering, Mahidol University, Salaya, Nakorn Pathom, 73170, Thailand \\ ${ }^{2}$ Laboratory of Computer Mechanics for Design (LCMD), Department of Mechanical Engineering, Faculty of Engineering, Mahidol University, \\ Salaya, Nakorn Pathom, 73170, Thailand
}

\begin{abstract}
Finite element model of tire rolling resistance test on the drum was developed using 3D steady state rolling analysis coupling with pre-inflation of 2D axisymmetric tire analysis. The complex components of the radial tires composing tread, sidewall, ply layers, steel belts, and lead wires were modeled using rebar elements which were embed into the rubber element using the tying equation. The Mooney-Rivlin hyperelastic constitutive model was employed to describe the large deformation behavior of tread and sidewall, while other components such as plies, steel belts and bead wires were assigned the linear isotropic material. The tire rolling resistance system was modeled by inflation of slick tire and compression on the drum for the footprint analysis regarding the rolling resistance test. The tire's steady state characteristics such as footprint contact pressure, rolling resistance force, and time response characteristic of tires were predicted instead the experiment of the prototype.
\end{abstract}

\section{Introduction}

The rolling resistance of a tire had been referred to the ratio between the energy loss per tire per travelling distance due to non-elastic deformation and energy dissipation of tires, while the rolling resistance coefficient was defined by the ratio of rolling resistance and the normal force on the tire [1]. The drum testing is an important method to measure the rolling resistance, which was performed by pressing an inflated tire against a drum with a desirable load. The drum was rotated and the tire's resistance was indirect to any variable such as force, torque, power, and deceleration [2]. The drum testing method had the advantage to reduce various factors from the vehicle suspension system and the pavement structure [3].

The finite element (FE) model of rolling resistance test was simple to run a tire model on a flat plate to reduce complexity caused by the rolling-contact on curved geometry but it was not agreed with the physical experiment caused by the curvature of the drum surface. The different in footprint and rolling characteristic became even more significant as the drum diameter get smaller [4]. In the recent years, FE models of the drum testing had been developed. The rotation system of drum and tire could be modeled by using transient dynamic analysis and steady state rolling (SSR) analysis which was based on the Eulerian and Lagrangian coupling [5]. Tönük [6] used kinematic inversion technique by assigning the drum to move around the fixed tire with same relative motion.
The transient dynamic analysis for the rolling resistance test of the pneumatic tire is preferred to use the explicit finite element method (FEM) to the implicit FEM. It is unnecessary if the SSR characteristic of the tire is considered. The transient dynamic method is disadvantaged because it requires many calculating times and induces cumulative error to solve the high nonlinear complexity of tire rotation and frictional rolling-contact mechanism. The transient dynamic FEM also tends to found limitation in dealing with rigid body rotation, forces users to model the drum with many fine meshes and caused to increase analysis time considerably. Particularly, the transient dynamic analysis will have severe disadvantage for modeling each component of tire for the high prediction accuracy. There was difficult to restore data for analysis due to the high amount of simulation result happened.

The SSR method had more advantages than the transient dynamic analysis because it required less analysis time and resource. The analysis time using may further reduce by refining of element concentration only in the critical contact region between tire and drum. This research aimed to develop a simulation model of the slick tire based on SSR analysis. The tire components were modeled with rebar elements and the tying equation to tie degree of freedom between tire's rubber and component elements. The axisymmetric analysis of the tire inflation was performed and then the results were used for the initial condition of rolling analysis.

\section{Hyperelastic material model}


The nonlinear large deformation behavior of the rubber material which was used to produce radial tires is governed by the Mooney-Rivlin hyperelastic constitutive model [7] as given by:

$$
W=W\left(\lambda_{1}, \lambda_{2}, \lambda_{3}\right)
$$

where $W$ is the strain energy density function, $\lambda$ is the stretch ratio.

Strain energy density function may be derived in the form of strain invariant (I) of left Cauchy-Green deformation tensor [8] is written by:

$$
W=W\left(I_{1}, I_{2}, I_{3}\right)
$$

where $I_{1}, I_{2}$ and $I_{3}$ are first, second and third strain invariant, respectively. The relation between strain invariant and stretch ratio can be described by:

$$
\begin{gathered}
I_{1}=\lambda_{1}^{2}+\lambda_{2}^{2}+\lambda_{3}^{2} \\
I_{2}=\lambda_{1}^{2} \lambda_{2}^{2}+\lambda_{2}^{2} \lambda_{3}^{2}+\lambda_{1}^{2} \lambda_{3}^{2} \\
I_{3}=\lambda_{1}^{2} \lambda_{2}^{2} \lambda_{3}^{2}
\end{gathered}
$$

Polynomial hyperelastic model is phenomenological model which based on strain energy in the form of polynomial equation of left Cauchy-Green deformation tensor as shown by:

$$
W=\sum_{i+j+k=1}^{N} C_{i j k}\left(I_{1}-3\right)^{i}\left(I_{2}-3\right)^{j}\left(I_{3}-3\right)^{k}
$$

where $C_{i j k}$ is material coefficients.

For an incompressible material, it can be simplified to:

$$
W=\sum_{i+j=1}^{N} C_{i j}\left(I_{1}-3\right)^{i}\left(I_{2}-3\right)^{j}
$$

Mooney-Rivlin hyperelastic model is the $1^{\text {st }}$ order polynomial hyperelastic model or $\mathrm{N}$ which equals to 1 . Mooney-Rivlin model can be used to reasonably predict stress-strain relationship in a small range of strain. The model can also be used to model nonlinear deformation behavior of tire during rolling. Mooney-Rivlin model may be written as:

$$
W=C_{10}\left(I_{1}-3\right)+C_{01}\left(I_{2}-3\right)
$$

where $C_{10}$ and $C_{01}$ is the material coefficient.

\section{Finite element modeling}

The FE model of tires under the rolling resistance test was performed by using commercial software, MSC. Marc. The slick tire shape like Michelin Agilis 195R14C tire was referred to generate finite element (FE) model for the rolling resistance test. The modelling consisted of two states: (1) the 2D inflation of tire using the axisymmetric analysis and (2) the $3 \mathrm{D}$ rolling resistance test analysis.

During the inflation state of the tire, the axisymmetric analysis was used to simplify the process. The slick tire was modeled in the 2D cross section which composed several components. The tread, sidewall, apex and bead wires are modeled by $2 \mathrm{D}$ quadratic axisymmetric elements while plies layers and steel belts are modeled by
1D rebar elements with independently assign the thickness in each layer (Fig. 1). Since the 2D and 1D element were meshed independently, nodes of the 2D and 1D element didn't share location with each other. In order to connect both meshes, the tying equation was used to tie degree of freedom between two types of elements. Table 1 describes types and the number of elements which are combined to be the axisymmetric FE model of radial tire.

The 3D radial tire model was then modeled by revolving the $2 \mathrm{D}$ axisymmetric model around its rotating axis or $\mathrm{x}$-axis. The $2 \mathrm{D}$ axisymmetric elements and 1D rebar elements were expanded to $3 \mathrm{D}$ continuum element and 2D rebar elements, respectively. The expanded element was uniformly divided into 20 sectors, which elements in the potential contacted region were divided with finer mesh density. Fig. 2 illustrates the FE model of the radial tire.

The FE model for the rolling resistance test of a radial tire was developed. The drum was modeled by a rigid body surface with a diameter of $1.7068 \mathrm{~m}$. The FE model of the radial tire was placed on a drum model. The drum model is assigned to be contacted with tread elements of

\begin{tabular}{|c|c|c|}
\hline $\begin{array}{l}\text { Radial tire } \\
\text { component }\end{array}$ & $\begin{array}{c}\text { Element type } \\
\text { (Axisymmetric/3D) }\end{array}$ & $\begin{array}{c}\text { Amount } \\
\text { (Axisymmetric/3D) }\end{array}$ \\
\hline Tread & Axisymmetric/Solid & $22 / 440$ \\
\hline Sidewall & Axisymmetric/Solid & $98 / 1,960$ \\
\hline Apex & Axisymmetric/Solid & $16 / 320$ \\
\hline Plies & 1D rebar/2D rebar & $88 / 1,760$ \\
\hline Steel belts ( $1^{\text {st }}$ layer $)$ & $1 \mathrm{D}$ rebar $/ 2 \mathrm{D}$ rebar & $17 / 340$ \\
\hline Steel belts ( $2^{\text {nd }}$ layer) & $1 \mathrm{D}$ rebar $/ 2 \mathrm{D}$ rebar & $17 / 340$ \\
\hline Cap ply & $1 \mathrm{D}$ rebar$/ 2 \mathrm{D}$ rebar & $32 / 640$ \\
\hline Bead wire & 1D rebar/2D rebar & $8 / 160$ \\
\hline
\end{tabular}
a tire model with a friction coefficient of 0.5 (Fig. 3). The Coulomb friction model using nonlinear arctangent smoothing function was assigned to model the contact friction between the radial tire and drum.

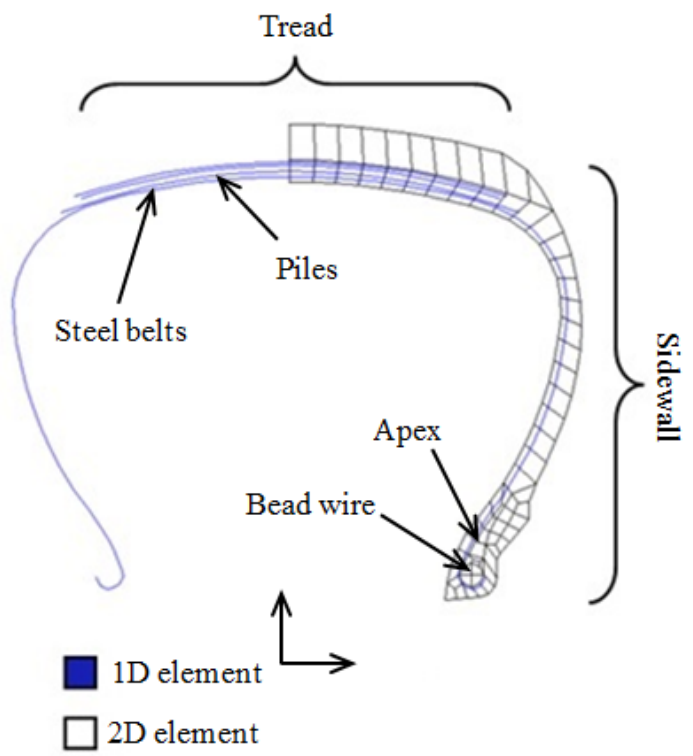

Figure 1. The cross section finite element model of a radial tire.

Table 1. Types and amount of elements for a radial tire model. 

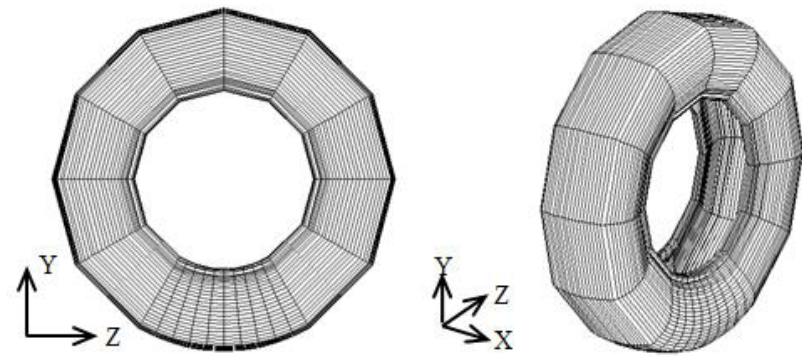

Figure 2. The 3D finite element of a radial tire.

The Mooney-Rivlin hyperelastic constitutive model was employed to tread, apex and sidewall of a tire model, while other components such as plies, steel belts and bead wires were defined as linear isotropic material. The material properties of radial tire components are shown in Table 2. These materials obtained by samples test according to the material testing standard.

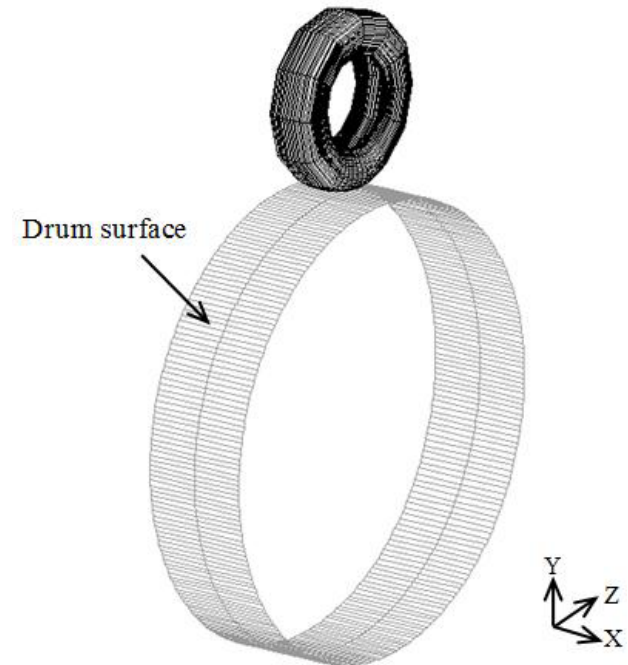

Figure 3. The FE model for the drum rolling resistance test of a radial tire.

Table 2. Mechanical properties of radial tire components.

\begin{tabular}{cccccc}
\hline \multirow{2}{*}{$\begin{array}{c}\text { Radial tire } \\
\text { component }\end{array}$} & \multirow{2}{*}{\begin{tabular}{c}
\multirow{2}{*}{ monstitutive } \\
model
\end{tabular}} & \multicolumn{5}{c}{ Mechanical property (MPa) } \\
& & C10 & C01 & E & $\boldsymbol{v}$ \\
\hline Tread & Hyperelastic & 0.003940 & 0.862791 & - & - \\
Sidewall & Hyperelastic & 0.152081 & 0.555920 & - & - \\
Apex & Hyperelastic & 0.645362 & 0.873692 & - & - \\
Plies & Linear & - & - & 3,800 & 0.48 \\
Steel belts & Linear & - & - & 110,316 & 0.46 \\
Cap ply & Linear & - & - & 3,800 & 0.48 \\
Bead wire & Linear & - & - & 200,000 & 0.30
\end{tabular}

Note $\mathrm{E}$ is elastic modulus, $v$ is Poisson's ratio, $\mathrm{C} 10$ and $\mathrm{C} 01$ is constant.

The $2 \mathrm{D}$ axisymmetric model of the tire was then used to analyze tire stress, strain, and deformation during inflation state. The boundary conditions consisted pressure of $0.45 \mathrm{MPa}$ and the fixed constraint were applied to the inside edge of tire element and nodes at the rim location, respectively. The $2 \mathrm{D}$ axisymmetric analysis results which consisted of stress, strain and deformation were collected and used to assign the initial condition of the next corresponding $3 \mathrm{D}$ rolling resistance analysis.
During rolling resistance analysis, the drum was brought to contact with the tire and the load of $807.5 \mathrm{~kg}$ was applied to the drum for the footprint and contact pressure prediction. In addition, the drum surface model was assigned to rotate about its center with a rotation velocity of $80 \mathrm{~km} / \mathrm{hr}$ after load and displacement were successfully assigned. The pressure applied into the tire was held constant during this state.

The FEA of the tire rolling resistance test on the drum was performed using personal computer Core-i5 CPU and 4GB RAM memory. The static and steady state analysis was used to solve for the $2 \mathrm{D}$ axisymmetric and 3D model respectively.

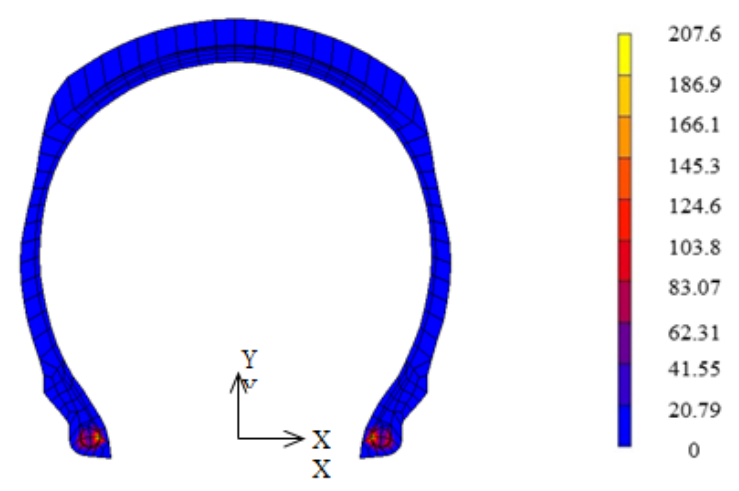

Figure 4. The inflation analysis results of the $2 \mathrm{D}$ axisymmetric tire model.

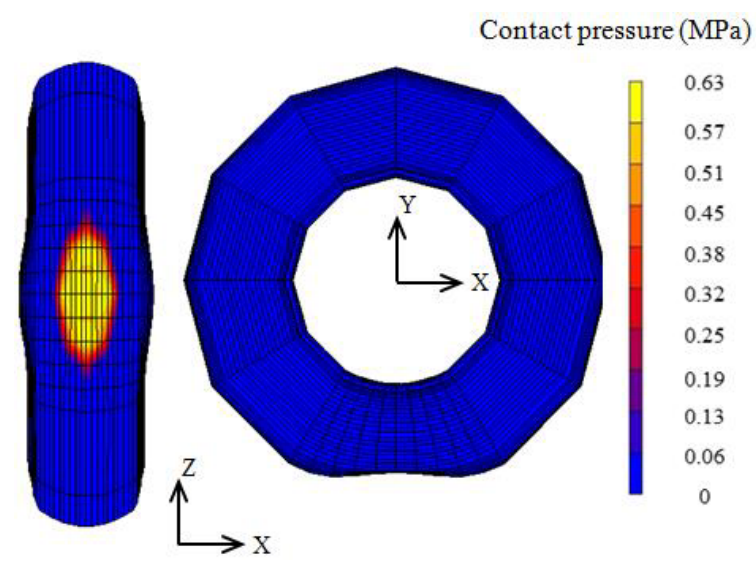

Figure 5. The contact pressure of the footprint analysis results.

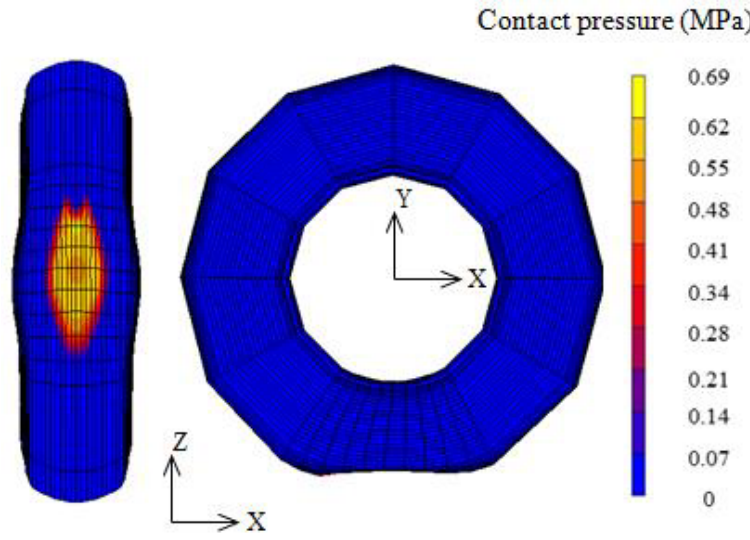

Figure 6. The footprint analysis results during steady state rolling. 


\section{Results and discussion}

The analysis results of the 2D axisymmetric model which compose deformation and stress during tire inflation state are illustrated in Fig. 4. The color contour was shown the maximum result in red and the minimum result in blue. These results were used as initial conditions of the 3D analysis and then the inflated 3D tire model was brought to contact with the drum and the y-direction force of $8,075 \mathrm{~N}$. The $3 \mathrm{D}$ footprint analysis results show the contact pressure distribution and deformation at loaded of $8,075 \mathrm{~N}$ in Fig. 5. After loading state, the SSR analysis was performed to inflate the $3 \mathrm{D}$ tire model for contacting with the drum. The drum surface at the contacted position was assigned to equivalently move with the speed of $22.22 \mathrm{~m} / \mathrm{sec}$ while the tire was assigned to rotate with spinning velocity of $66.6126 \mathrm{rad} / \mathrm{sec}$, which corresponded to traveling speed at $80 \mathrm{~km} / \mathrm{hr}$. The contact pressure distribution and deformation during rolling at steady state are shown in Fig. 6. The sidewall of tire was observed to slightly bulge during footprint analysis and continue to be bulged during rolling. The contact pressure showed an effective area of the tire which contacted on the drum or the road surface in the general usage, therefore, it could be used to indicate how well various tire's parameters such as the tire shape, material, components, pressure, tread pattern and depth effect traction of the vehicle. The rolling resistance force was also calculated from the reaction force between the drum and tire. The rolling resistance force and drum loading are plotted by graphs as shown in Fig. 7. The graphs were divided into three sections upon the time during three states of the 3D analysis which were tire's moving into contact state, drum loading state, and SSR. The force by drum and rolling resistance were satisfied the condition of drum test. The resistance force increased after the inflated tire was loaded with the drum force.

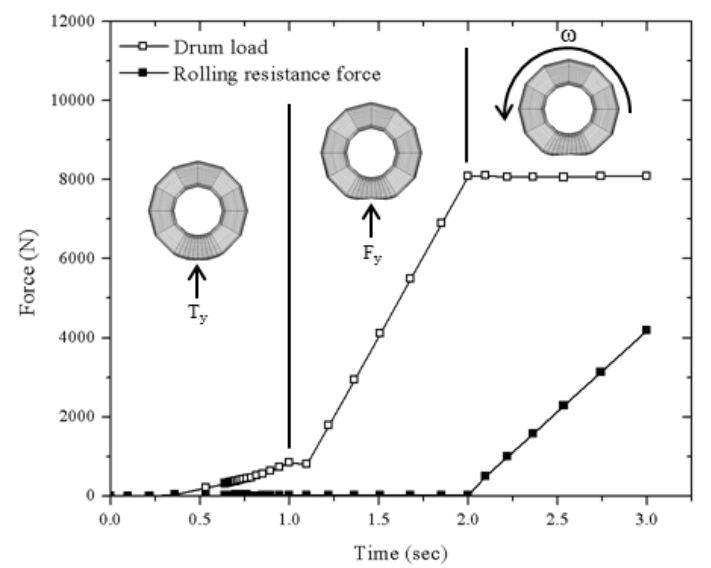

Figure 7. The applied drum load and the rolling resistance force vs. time.

\section{Conclusions}

The FE model of the tire rolling resistance with drum testing method was developed based on the 2D axisymmetric and the 3D SSR analysis. The MooneyRivlin hyperelastic constitutive model was used to describe the nonlinear large deformation behavior of the tire, while other components such as plies, steel belts and bead wires were defined as linear isotropic material. The tire components were modeled with rebar elements and tied to rubber elements which had a different degree of freedom using the tying equation. The $2 \mathrm{D}$ axisymmetric elements were expanded to the $3 \mathrm{D}$ elements by revolving around the tire axis. The $2 \mathrm{D}$ axisymmetric analysis results of the tire during inflation state were used as the initial condition of 3D analysis. The inflated 3D tire model was brought for contacting with the imported drum surface. The drum was loaded and SSR analysis was performed. The analysis results showed that FE model of the tire using the 3D SSR analysis combining with the pre-load of the 2D axisymmetric analysis and the embed rebar element could be used to predict steady state characteristics such as stress, deformation, contact pressure, and rolling resistance force for the drum testing. This technique, there are more advantages, less analysis time and resource requirement than the previous 3D transient dynamic analysis. Finally, this FEM could plentifully give the useful information and understanding for designing and development the radial tires in the near future.

\section{References}

1. L.G. Andersen, J.K. Larsen, E.S. Fraser, B. Schmidt, J.C. Dyre, J. Transp. Eng. 141, 1 (2015)

2. International Organization for Standardization. ISO 18164:2005 Passenger car, truck, bus and motorcycle tyres-Methods of measuring rolling resistance (ISO, Geneva, 2005)

3. H.W. Lee, J.R. Cho, W.B. Jeong, K.M. Jeong, K.W. Kim, Int. J. Automot. Techn. 15, 411 (2014)

4. N. Korunović, M. Trajanović, M. Stojković, D. Mišić, J. Milovanovićet, J. Mech. Eng. 57, 888 (2011)

5. R. Behnke, M. Kaliske, Int. J. Nonlinear Mech. 68, $101(2015)$

6. E. Tönük, Y. S. Ünlüsoy. Computers \& Structures 79, 1219 (2001)

7. G.T. Mase, R.E. Smelser, G.E. Mase, Continuum Mechanics for Engineer (CRC press, Florida, 2010)

8. G.A. Holzapfel, Nonlinear Solid Mechanics (John Wiley \& Sons, England, (2000) 